\title{
The mass-velocity and intensity-velocity relations in jet-driven molecular outflows
}

\author{
T. P. Downes ${ }^{1}$ and S. Cabrit ${ }^{2}$ \\ ${ }^{1}$ School of Mathematical Sciences, Dublin City University, Dublin 9, Ireland \\ 2 LERMA, Observatoire de Paris, 61 Av. de l'Observatoire, 75014 Paris, France \\ Received 18 June 2001 / Accepted 12 March 2003

\begin{abstract}
We use numerical simulations to examine the mass-velocity and intensity-velocity relations in the $\mathrm{CO} J=2-1$ and $\mathrm{H}_{2} \mathrm{~S}(1) 1-0$ lines for jet-driven molecular outflows. Contrary to previous expectations, we find that the mass-velocity relation for the swept-up gas is a single power-law, with a shallow slope $\simeq-1.5$ and no break to a steeper slope at high velocities. An analytic bowshock model with no post-shock mixing is shown to reproduce this behaviour very well.

We show that molecular dissociation and the temperature dependence of the line emissivity are both critical in defining the shape of the line profiles at velocities above $\sim 20 \mathrm{~km} \mathrm{~s}^{-1}$. In particular, the simulated CO $J=2-1$ intensity-velocity relation does show a break in slope, even though the underlying mass distribution does not. These predicted CO profiles are found to compare remarkably well with observations of molecular outflows, both in terms of the slopes at low and high velocities and in terms of the range of break velocities at which the change in slope occurs. Shallower slopes are predicted at high velocity in higher excitation lines, such as $\mathrm{H}_{2} \mathrm{~S}(1) 1-0$.

This work indicates that, in jet-driven outflows, the CO $J=2-1$ intensity profile reflects the slope of the underlying massvelocity distribution only at velocities $\leq 20 \mathrm{~km} \mathrm{~s}^{-1}$, and that higher temperature tracers are required to probe the mass distribution at higher speed.
\end{abstract}

Key words. hydrodynamics - shock waves - ISM: jets and outflows - ISM: molecules

\section{Introduction}

Various authors have noted that the intensity-velocity relationship observed in low- $J$ CO lines in molecular outflows tends to follow a broken power-law $I_{\mathrm{CO}}(v) \propto v^{-\gamma}$, with $\gamma \approx 1.8 \pm 0.5$ up to line-of-sight velocities $v_{\text {break }} \approx 10-30 \mathrm{~km} \mathrm{~s}^{-1}$ and $\gamma \approx 3-7$ at higher velocities (e.g. Rodríguez et al. 1982; Stahler 1994; Bachiller \& Tafalla 1999; Richer et al. 2000). This property is an important test for proposed mechanisms of molecular outflow acceleration. In particular, recent work has addressed this issue in the case of entrainment by a jet.

Using an analytic model of a jet/bowshock system, Zhang $\&$ Zheng (1997) predicted a mass distribution, $m(v)$, following a broken power-law $v^{-\mu}$ with slopes $\mu \approx 1.8$ up to $10 \mathrm{~km} \mathrm{~s}^{-1}$ and $\mu \approx 5.6$ beyond. The observed values of $\gamma$ in CO intensity profiles are then reproduced, provided the $\mathrm{CO}$ abundance and line excitation do not vary much with velocity. However, this seems highly unlikely, given the broad range of shock strengths in a bowshock.

Smith et al. (1997) conducted numerical simulations of jetdriven molecular outflows that take into account dissociation

Send offprint requests to: T. P. Downes, e-mail: turlough.downes@dcu.ie

Correspondence to: School of Mathematical Sciences, Dublin City University, Glasnevin, Dublin 9, Ireland. and heating in shocks. They find that $I_{\mathrm{CO}}(v)$ follows a powerlaw $v^{-\gamma}$ with $\gamma \approx 1.2-1.6$ up to $10 \mathrm{~km} \mathrm{~s}^{-1}$, and a steeper slope further out, in qualitative agreement with observations. They attribute this result to a much steeper mass-velocity relation, $m(v) \propto v^{-3.5}$, than Zhang \& Zheng (1997). However, their reasoning involves an erroneous high-temperature dependence of the CO emissivity ( $\propto T$ instead of $T^{-1}$ ). Therefore, the actual origin of the slope of $I_{\mathrm{CO}}(v)$ in jet simulations, and the underlying mass-velocity relation itself, remain to be clearly established. In this work we clarify this issue using simulations at higher resolution, and analytical modelling.

\section{Numerical method}

The code used in this work is very similar to that used in Downes \& Ray (1999). The initial conditions are also very similar, but we give a brief overview of them here for completeness.

The simulations are performed in 2D cylindrical symmetry. The densities of molecular and atomic hydrogen are tracked, along with the ionisation fraction of hydrogen. The $\mathrm{CO}$ density is assumed to be a constant $\left(10^{-4}\right)$ fraction of the $\mathrm{H}_{2}$ density by number. The numerical scheme is a Godunov scheme which is second order in time and space (see, e.g., Downes \& Ray 1999). 


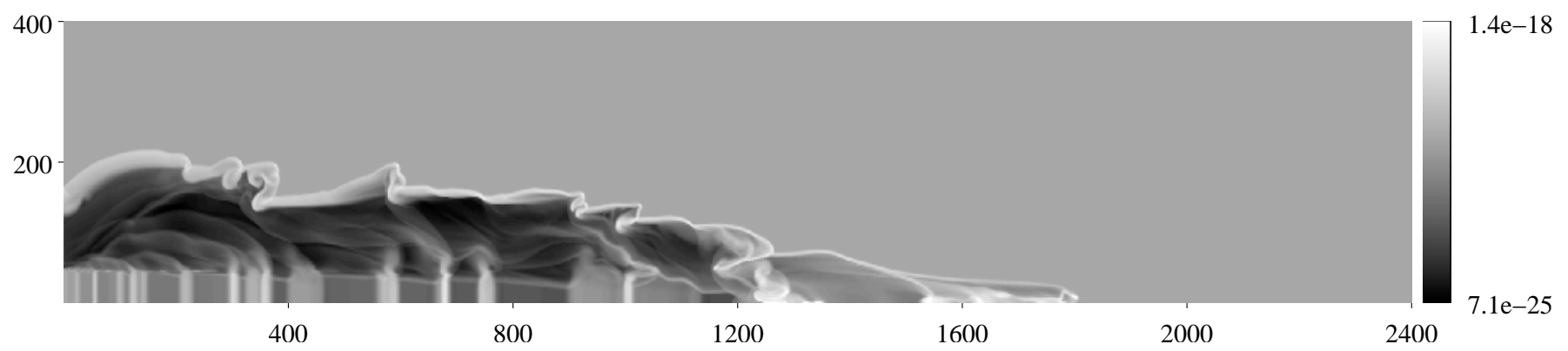

Fig. 1. Log-scale plot of the distribution of number density for the pulsed jet with $\frac{v_{1}}{v_{0}}=0.6$ at $t=400 \mathrm{yrs}$. The density scale is in units of $\mathrm{g} \mathrm{cm}{ }^{-3}$ while the distance scales are in units of $1 \times 10^{14} \mathrm{~cm}$.

The grid-spacing was set at $10^{14} \mathrm{~cm}$. The jet radius was $5 \times 10^{15} \mathrm{~cm}$, and its density was $100 \mathrm{~cm}^{-3}$ (equal to the ambient density), thus allowing a resolution of cooling layers a factor of $10^{2}-10^{4}$ higher than in Smith et al. (1997). The time-averaged jet velocity, $v_{0}$, was $215 \mathrm{~km} \mathrm{~s}^{-1}$. Superimposed on this were sinusoidal variations with periods of $5,10,20$ and 50 years with total amplitude $v_{1}$.

In order to explore the effects of varying the jet velocity, three simulations were run, as follows: (1) a "steady" jet where $\frac{v_{1}}{v_{0}}=0$; (2) a "pulsed" jet where $\frac{v_{1}}{v_{0}}=0.6$; and (3) a "rampedup" jet where $\frac{v_{1}}{v_{0}}=0.6$ and $v_{0}$ increases linearly in time from 0 to $215 \mathrm{~km} \mathrm{~s}^{-1}$ over 100 years. Typically, simulations of jets start off with the jet propagating at full speed into the ambient medium. It is likely that YSO jets do not "switch on" impulsively, and it has been suggested recently (Lim et al. 2002) that a slow start-up of a jet may lead to increased molecular abundance at the head of the bowshock, which would then affect the resulting intensity-velocity relation that we wish to model.

\section{Numerical results}

In this section, we concentrate on the results of simulation (2) (pulsed jet) described in Sect. 2, at a time $t=400$ yrs. Results for other times and for the steady and ramped-up jets (simulations (1) and (3) in Sect. 2) will be discussed in Sect. 5.

Figure 1 contains a log-scale plot of the density distribution for the chosen simulation. We can see that the bowshock is rather irregular. These irregularities are attributed to the growth of the Vishniac instability (Downes \& Ray 1999). In addition, several "mini-bowshocks" are present along the jet length, tracing internal working surfaces resulting from the variability of the jet velocity. Figure 2 contains plots of various mass-velocity and intensity-velocity relations for the same simulation, assuming an inclination of $30^{\circ}$ to the plane of the sky. We discuss each of these distributions in detail in the following.

\subsection{The total mass-velocity relation}

A significant feature of Fig. 2 is that $m(v)$, the mass-velocity relation for all swept-up material (i.e. excluding jet material), remains quite shallow across the whole velocity range. It is essentially flat at the lowest velocities $\left(v<3 \mathrm{~km} \mathrm{~s}^{-1}\right)$, then follows an approximate power-law $m(v) \propto v^{-\mu}$ with exponent $\mu \simeq 1.5$ at intermediate velocities, before rising slightly again

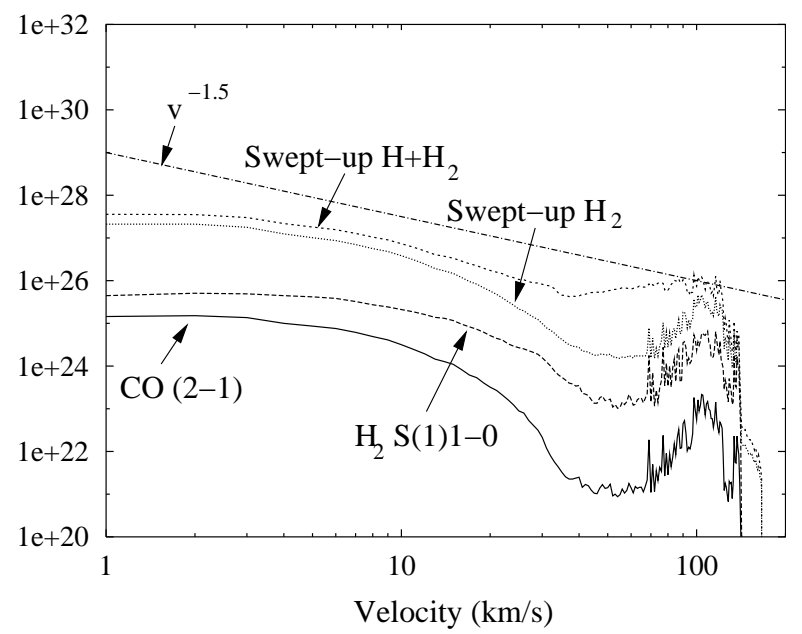

Fig. 2. Plots of the mass-velocity relations for all swept-up material (i.e. excluding jet material), $m(v)$, and for molecular swept-up material only, $m_{\mathrm{H}_{2}}(v)$, for the pulsed jet at $t=400$ yrs. Also shown are the intensity-velocity relations for the CO $J=2-1$ line and for the $\mathrm{H}_{2} \mathrm{~S}(1) 1-0$ line (arbitrary vertical offsets). An angle of $30^{\circ}$ to the plane of the sky is assumed.

above $v \simeq 40 \mathrm{~km} \mathrm{~s}^{-1}$. The power-law slope that we find agrees quite well with the value of $\mu \simeq 1.8$ predicted by the analytical model of Zhang \& Zheng (1997) for low velocities, but their predicted break to a steeper slope $\mu \simeq 5$.6 at velocities above $10 \mathrm{~km} \mathrm{~s}^{-1}$ is not seen.

\subsection{The molecular mass-velocity relation}

The mass-velocity relation for swept-up molecular material, $m_{\mathrm{H}_{2}}(v)$, behaves similarly to the total mass-velocity relation, $m(v)$, at low velocities. It does, however, become considerably steeper than $m(v)$ at higher velocities $\left(v \geq 20 \mathrm{~km} \mathrm{~s}^{-1}\right)$. This steepening occurs because material at these velocities has been accelerated by faster shocks near the apex of the bowshock, where significant molecular dissociation occurs. Indeed, the typical dissociation limit for low-density non-magnetic shocks is $\simeq 30 \mathrm{~km} \mathrm{~s}^{-1}$ (see e.g. Flower et al. 2003). We note that the molecular fraction appears to rise again at the highest velocities (above $80 \mathrm{~km} \mathrm{~s}^{-1}$ ). This is an unavoidable result of numerical diffusion at the bow head, which results in mixing of molecular jet material into the high-velocity swept-up gas. 


\subsection{The $I_{C O}(v)$ relation}

The CO $J=2-1$ line emissivity at each grid point of the simulation was calculated from the local density and temperature according to the analytical formulae of McKee et al. (1982), which take into account sub-thermalization of the levels. Emission was assumed optically thin, which is probably a good approximation for velocities above a few $\mathrm{km} \mathrm{s}^{-1}$.

Figure 2 shows that the resulting intensity-velocity relation for the $\mathrm{CO} J=2-1$ line, $I_{\mathrm{CO}}(v)$, follows the same slope as $m_{\mathrm{H}_{2}}(v)$ at low velocities, but breaks to an even steeper relation than $m_{\mathrm{H}_{2}}(v)$ at velocities above $\simeq 30 \mathrm{~km} \mathrm{~s}^{-1}$. This steepening results from the temperature dependence of the $\mathrm{CO}(2-1)$ emissivity per molecule. This dependence (assuming LTE) is given in terms of a function $\Omega(T)$ in Cabrit \& Bertout (1990), and is plotted as a function of temperature for the CO $J=2-1$ line in Fig. 13 of Lada \& Fich (1996). The line emissivity per molecule is seen to initially increase steeply with $T$ up to a maximum at $T \simeq 20 \mathrm{~K}$, similar to the excitation energy of the upper level of the transition. For higher temperatures, however, it then decreases as $T^{-1}$, due to the larger number of energy levels available to the molecule (or equivalently, as an effect of the partition function). Hence, as we go to higher velocities, not only are there fewer molecules to emit (due to shock dissociation), but they are hotter (because they have been through a stronger shock) and so they emit less efficiently in the CO $J=2-1$ line. This produces an even steeper slope at high velocities in $I_{\mathrm{CO}}(v)$ than in $m_{\mathrm{H}_{2}}(v)$.

\subsection{The $I_{H_{2}}(v)$ relation}

It is interesting to explore the predicted intensity-velocity relation for the $\mathrm{H}_{2} \mathrm{~S}(1)$ 1-0 line, $I_{\mathrm{H}_{2}}(v)$, as it has been recently observed in a few flows (Salas \& Crúz-González 2002) and the line is of much higher excitation than the CO $J=2-1$ line. Such predictions from jet simulations have, to the best of our knowledge, never been presented so far. For simplicity, we computed the line emissivity assuming LTE. Although this is not fully justified at the moderate densities of our simulations, it is sufficient to illustrate the change of slope with excitation of the line that we predict to occur in jet-driven outflows.

From Fig. 2 we see that, unlike the CO 2-1 line, this intensity-velocity relation is shallower than $m_{\mathrm{H}_{2}}(v)$ up to $30 \mathrm{~km} \mathrm{~s}^{-1}$, and therefore does not show as dramatic a break in slope at high velocities. This is due to the fact that the the upper energy level for the $\mathrm{H}_{2} \mathrm{~S}(1) 1-0$ line lies at $7000 \mathrm{~K}$; hence the emissivity per molecule is still increasing with $T$ up to temperatures of $\simeq 10^{4} \mathrm{~K}$, so that higher velocity, hotter material now emits more efficiently in the $\mathrm{H}_{2} \mathrm{~S}(1) 1-0$ line. The $I_{\mathrm{H}_{2}}(v)$ distribution is then shallower than the underlying mass distribution.

\section{Comparison with observations}

In this section, we compare our simulation results directly with observations. Figure 3 contains plots of our results for the $m(v)$ and $I_{\mathrm{CO}}(v)$ relations, along with observed intensity-velocity relations in $\mathrm{CO} J=2-1$ for various bipolar outflows (taken from Bachiller \& Tafalla 1999). A similar comparison with the

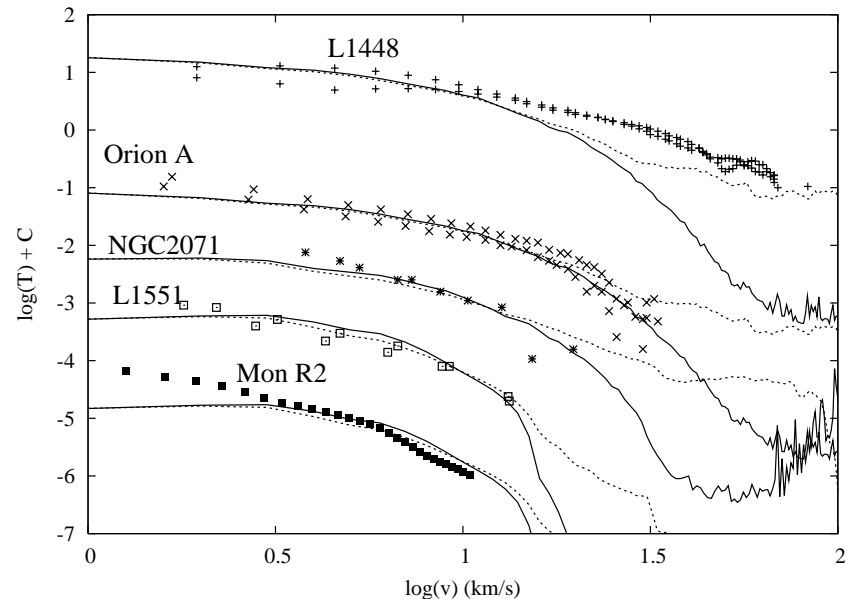

Fig. 3. Plots of the observed intensity-velocity relations for L1448, Orion A, NGC 2071, L1551 and Mon R2 (“+” signs, crosses, stars, open boxes, and filled boxes respectively). Also shown are the simulated $m(v)$ and $I_{\mathrm{CO}}(v)$ (dotted and solid lines, respectively, with arbitrary vertical offsets) for comparison. The fits to both L1448 and Orion A assume an angle of $60^{\circ}$ to the plane of the sky, for NGC 2071 the angle is assumed to be $30^{\circ}$, and for L1551 and Mon R2 the flows are assumed to be in the plane of the sky.

results of Salas \& Crúz-González (2002) will be made in a forthcoming paper, using NLTE calculations of the $\mathrm{H}_{2} \mathrm{~S}(1) 1-$ 0 line intensity.

It is notable that the simulation results for the $I_{\mathrm{CO}}(v)$ relation reproduce the observations remarkably well, given that the velocity of the jet was arbitrarily chosen and the simulation timescale is shorter than that of the physical systems (estimated ages range from $1000 \mathrm{yrs}$ in Orion to several times $10^{4} \mathrm{yrs}$ for L 1551 and Mon R2). It is also interesting to note that the predicted $I_{\mathrm{CO}}(v)$ more closely matches the observations than the total mass-velocity relation $m(v)$, save for L1448.

The only parameters which have been tuned in this comparison are the vertical scale (i.e. a scaling in the ambient gas density), and the assumed inclination to the plane of the sky. The latter parameter can be seen to have a sizeable effect on the break velocity. While our adopted inclinations do not exactly match that inferred from observations for each of the systems shown, the trend is correct in the sense that Orion A and L1448 are the most inclined to the plane of the sky, NGC 2071 is intermediate, and L1551 and Mon R2 are believed to be closest to the plane of the sky.

In summary, it seems clear, on the basis of these simulations, that a jet-driven bowshock can reproduce the observed $I_{\mathrm{CO}}(v)$ remarkably well, both in terms of the slopes at low and high velocities and in terms of the range of break velocities where this change of slope occurs, without the need to invoke two distinct entrainment mechanisms as is done in Zhang \& Zheng (1997).

The simulation results do tend to be slightly too flat at the lowest velocities, especially in the oldest flows (Mon R2 in particular). This could be due to the short timescales in our simulations, as we now show. 


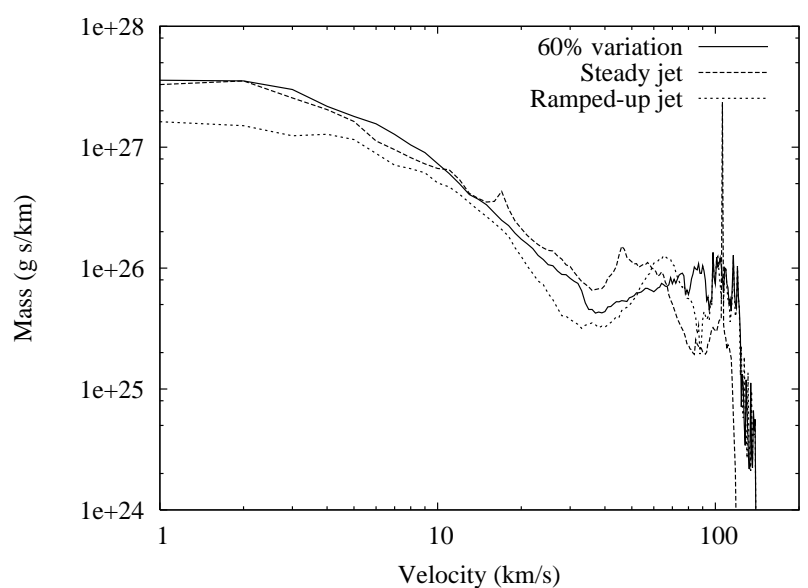

Fig. 4. Plots of the mass-velocity distribution of swept-up material for three simulations with differing jet velocity variability at $t=400 \mathrm{yrs}$, assuming an angle of $30^{\circ}$ to the plane of the sky. Note the similarity between all three relations.

\section{The effect of age and time-variability}

\subsection{Effect of time-variability of the jet velocity}

It is important to verify that our results are not critically dependent upon the adopted jet velocity behaviour. Figure 4 compares the $m(v)$ relation for the pulsed jet with the swept-up mass-velocity relation for the other two simulations (steady jet and ramped-up jet), at the same time and same inclination. It is clear that the relations are very similar. This is also the case for the $m_{\mathrm{H}_{2}}(v), I_{\mathrm{CO}}(v)$ and $I_{\mathrm{H}_{2}}(v)$ relations (not shown). This strongly suggests that variability of the jet velocity (at least on time-scales less than about 50 yrs) will not greatly affect the resulting mass-velocity or intensity-velocity relations.

\subsection{Effect of age}

It is also important to verify that our results do not depend critically on the simulation timescale, since observed flows cover a large range of ages. Figure 5 contains plots of the mass-velocity relation for the pulsed jet simulation (2) at different times from $t=100-400$ yrs. It can be seen that the part of the $m(v)$ distribution that follows a power-law $\propto v^{-1.5}$ extends to progressively lower velocities as time goes, while the flat portion of $m(v)$ at very low velocity shrinks. This behaviour can be understood as follows: the power-law regime corresponds to the part of the bow structure presently on the grid, while the flat part of $m(v)$ comes from the truncation of the bow at the left border of the computational grid; as the bow head advances, more of the bow structure appears on the grid, so that the part affected by truncation is at a lower speed (since velocity decreases away from the bow head). Hence, we expect that for longer timescales, the flat part of $m(v)$ and $I_{\mathrm{CO}}(v)$ will fall to very low velocities, while the $v^{-1.5}$ power-law should extend over the whole observable velocity range, in better agreement with observations of old flows such as Mon R2. Simulations over very long timescales are under way to study this hypothesis.

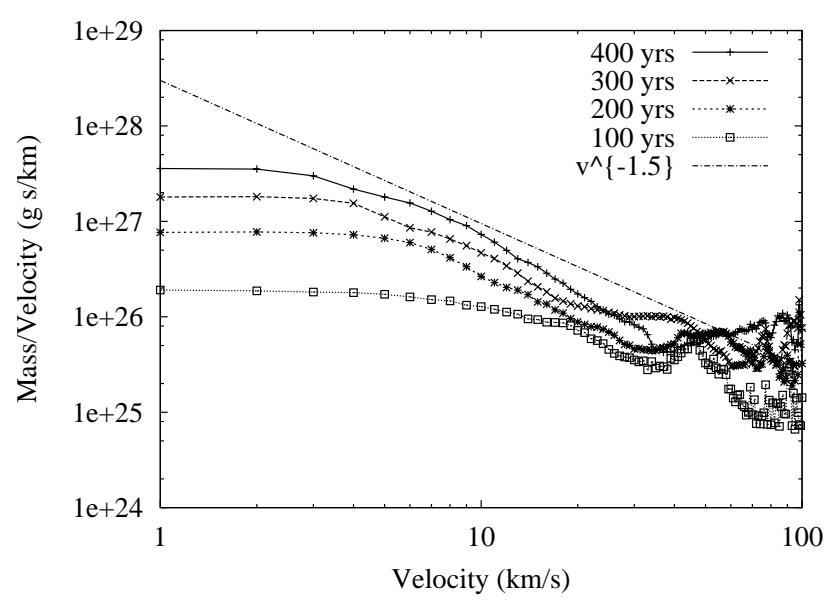

Fig. 5. Plots of the mass-velocity relation for all swept-up material in the pulsed jet (simulation (2)) at $t=100,200,300$ and $400 \mathrm{yrs}$, assuming an angle of $30^{\circ}$ to the plane of the sky.

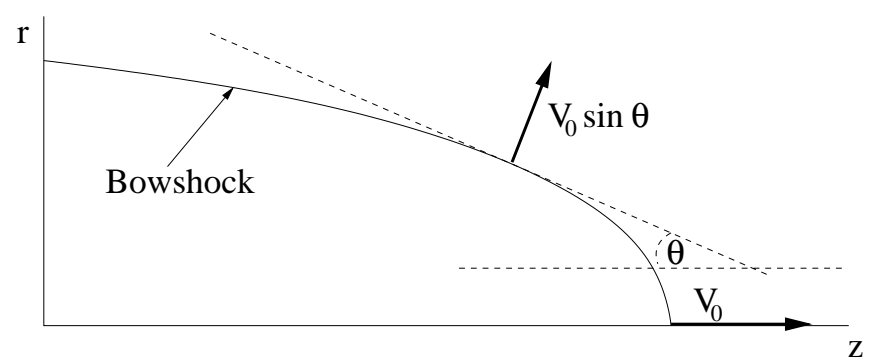

Fig. 6. Schematic diagram of the set-up used in the analytic calculations of the mass-velocity relation.

\section{Analytic model}

In this section we explore in more depth how a jet-driven bowshock can produce the power-law slope $\mu \simeq 1.5$ of the massvelocity relation discussed in Sect. 3.1. Figure 6 contains a schematic diagram of the set-up used in this discussion.

Consider an idealised bowshock, the shape of which is given by the relation $z \propto r^{s}$, moving in the positive $z$ direction with constant velocity $v_{0}$ into an ambient medium of constant density $\rho_{\mathrm{a}}$. We assume that there is no mixing in the bow, so that the magnitude of the post-shock velocity in the observer's frame is $v_{0} \sin \theta$, with $\tan \theta=\mathrm{d} r / \mathrm{d} z$ (see Fig. 6). An observer situated along the $z$-axis will then see a line-of-sight velocity of

$v=v_{0} \sin ^{2} \theta$.

Far from the apex, where $\theta$ is small, $v \propto \tan ^{2} \theta \propto\left(r^{2}\right)^{1-s}$. The rate of increase of mass at projected velocity $v$ is then

$\frac{\mathrm{d}}{\mathrm{d} t}(m(v) \mathrm{d} v)=\rho_{\mathrm{a}} v_{0} 2 \pi r(v) \mathrm{d} r \propto(v)^{s /(1-s)} \mathrm{d} v$.

Hence the mass-velocity relation in this case is a power-law

$m(v) \propto v^{-\mu} \quad$ with $\quad \mu=\frac{s}{s-1}$.

In order to verify that the no-mixing model can indeed explain the $m(v)$ relations found in our simulations, we need to determine the appropriate value of $s$ for the simulated bows. For this, we examine the rate at which ambient mass is swept up by the 


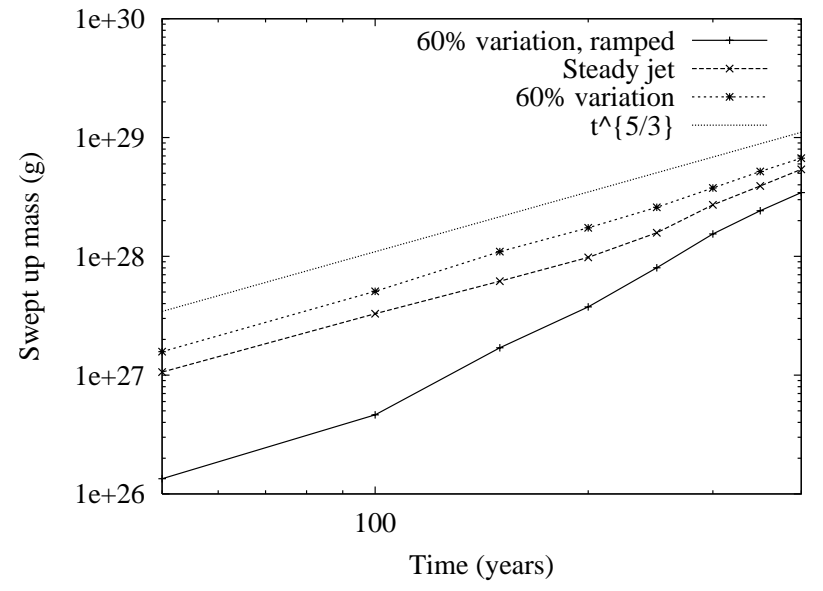

Fig. 7. Plots of the total swept-up mass against time for each of the simulations.

bowshock, integrated over all velocities. For an adopted ideal bow shape $z \propto r^{s}$, this rate is

$\frac{\mathrm{d} m}{\mathrm{~d} t}(t)=\pi r_{0}^{2} \rho_{\mathrm{a}} v_{0}$

where $r_{0} \propto z^{1 / s}(t)$ is the maximum bowshock radius and $z(t)=v_{0} t$ is the length of the bowshock, both at time $t$. Hence, the total swept-up mass as a function of time is predicted to vary as

$m(t) \propto t^{\alpha} \quad$ with $\quad \alpha=1+2 / s$.

In Fig. 7 we plot the total swept-up mass $m(t)$ as a function of time for each of the simulations. Indeed, we find that it closely follows a power-law, with a slope $\alpha=1.7-1.8$. Note that the apparent deviation of simulation (3) from this law at early times is due to the initial ramping up of the jet velocity $v_{0}$. Since the bow model predicts $\alpha=1+2 / s$, we infer that the appropriate value of $s$ for our simulated bowshocks is $s=2.5-2.9$. If we now substitute this range of values of $s$ into Eq. (3), we find that our simple bow model with no mixing predicts an exponent of the mass-velocity relation in the range $\mu=1.5-1.7$. This is remarkably close to what we find in the simulations (Sect. 3.1). Note that the same bow model, but assuming instantaneous mixing of post-shock swept-up gas, predicts a much steeper slope $\mu=2+s / 2=3.5$ (Smith et al. 1997).

It is important to keep in mind that the power-law slope is predicted to hold only for small values of $\theta$, i.e. sufficiently far from the head of the bowshock. In Fig. 8 we plot the massvelocity relation for a theoretical bow with $s=3$, calculated numerically without the assumption of small $\theta$. The power-law of slope $\mu=s /(s-1)=1.5$ is seen to give a very good approximation to the actual $m(v)$ up to about $20 \%$ of the jet velocity. At higher velocities, the mass-velocity relation flattens out and then increases with velocity. This behaviour is clearly also seen in our simulated $m(v)$ (see Fig. 2), although we do not find as sharp a peak at $v_{0}$, due to the fact that the head of the bowshock is rather irregular.

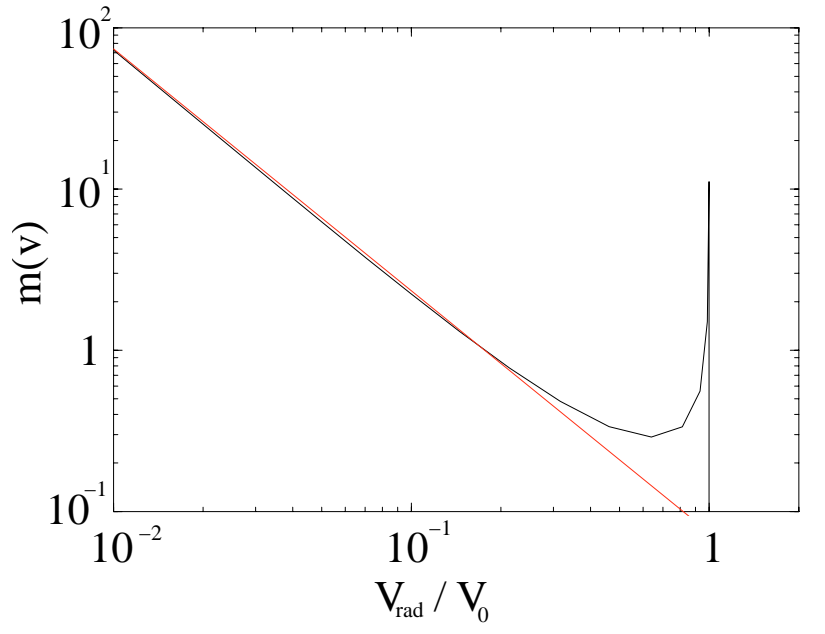

Fig. 8. Plot of the total swept-up mass as a function of velocity predicted for an ideal bowshock shape described by $z \propto r^{3}$ viewed poleon, with no post-shock mixing. The straight line is the power law of exponent -1.5 predicted for the bow wings (see text).

\section{Conclusions}

We find that the mass-velocity relation in simulations of jetdriven molecular outflows is a shallow power-law of exponent $\simeq-1.5$, with no break to a steeper slope at higher velocity, unlike previous analytical predictions by Zhang \& Zheng (1997). This $m(v)$ relation can be well explained by a bowshock model with no post-shock mixing. It does not appear to be consistent with the full mixing model proposed by Smith et al. (1997).

We also find that the resulting intensity-velocity relation for the CO $J=2-1$ line compares remarkably well with observations of molecular outflows. In particular, it does have a break in slope around $20-30 \mathrm{~km} \mathrm{~s}^{-1}$. The break is found to result from molecular dissociation near the bow apex, and from the $1 / T$-dependence of emission at temperatures exceeding the energy of the upper level of the line. Because of this dependence on $T$, a jet-driven model predicts a shallower slope at high velocity in higher excitation lines (e.g. $\mathrm{H}_{2} \mathrm{~S}(1)(1-0)$ and high- $J$ CO lines), which could be tested by ongoing studies. Another implication of our results is that, in jet-driven outflows, the CO $J=2-1$ intensity profile reflects the slope of the underlying mass-velocity distribution only at velocities $\leq 20 \mathrm{~km} \mathrm{~s}^{-1}$, and that higher temperature tracers are required to probe the mass distribution at higher speed.

The only weak point of a bowshock model with no mixing is that emitting gas expands perpendicular to the bow surface, and thus there is some difficulty in explaining the apparent "forward-directed" motion of molecular outflows noted by Lada \& Fich (1996). Quantitatively, our simulations predict comparable amounts of redshifted and blueshifted gas up to velocities of 3-4 $\mathrm{km} \mathrm{s}^{-1}$. Full mixing was invoked by Smith et al. (1997) to alleviate this problem, but we have shown here that it would predict a very steep $m(v)$ of slope $\simeq-3.5$ and an even steeper CO intensity-velocity relation (due to dissociation and temperature effects), inconsistent with observations. We note that another situation in which motion will be more 
forward-directed is when the bowshock propagates into already moving material. This is an interesting issue which clearly requires further work.

Acknowledgements. We would like to thank Rafael Bachiller for kindly providing us with the observational data in Fig. 3 and for stimulating discussions. We are indebted to Alex Raga for his contribution to the code calculating $\mathrm{CO}$ emissivities, and for helpful suggestions about this work. T. P. Downes acknowledges support as a visiting astronomer at the Observatoire de Paris.

\section{References}

Bachiller, R., \& Tafalla, M. 1999, in The Origin of Stars and Planetary Systems, ed. C. J. Lada, \& N. D. Kylafis (Kluwer Academic Publishers), 227

Cabrit, S., \& Bertout, C. 1990, ApJ, 348, 530
Downes, T. P., \& Ray, T. P. 1999, A\&A, 345, 977

Flower, D., et al. 2003, MNRAS, in press

Lada, C. J., \& Fich, M. 1996, ApJ, 459, 638

Lim, A. J., Raga, A. C., Rawlings, J. M. C., \& Williams, D. A. 2002, MNRAS, 335, 817

McKee, C. F., Storey, J. W. V., Watson, D. M., \& Green, S. 1982, A\&A, 113, 285

Richer, J. S., Shepherd, D. S., Cabrit, S., Bachiller, R., \& Churchwell, E. 2000, in Protostars and Planets IV, ed. V. Mannings, A. P. Boss, \& S. S. Russell (University of Arizona Press), 867

Rodríguez, L. F., Carral, P., Moran, J. M., \& Ho, P. T. P. 1982, ApJ, 260,635

Salas, L., \& Crúz-González, I. 2002, ApJ, 572, 227

Smith, M. D., Suttner, G., \& Yorke, H. W. 1997, A\&A, 323, 223

Stahler, S. 1994, ApJ, 422, 616

Zhang, Q., \& Zheng, X. 1997, ApJ, 474, 719 